\title{
On-chip patch antenna on InP substrate for short-range wireless communication at 140 GHz
}

Dong, Yunfeng; Johansen, Tom Keinicke; Zhurbenko, Vitaliy

\section{Published in:}

Proceedings of Microwave and Optoelectronics Conference (IMOC), 2017 SBMO/IEEE MTT-S International

Link to article, DOI:

10.1109/IMOC.2017.8121032

Publication date:

2017

Document Version

Peer reviewed version

Link back to DTU Orbit

Citation (APA):

Dong, Y., Johansen, T. K., \& Zhurbenko, V. (2017). On-chip patch antenna on InP substrate for short-range wireless communication at $140 \mathrm{GHz}$. In Proceedings of Microwave and Optoelectronics Conference (IMOC), 2017 SBMO/IEEE MTT-S International (pp. 1-5). IEEE. https://doi.org/10.1109/IMOC.2017.8121032

\section{General rights}

Copyright and moral rights for the publications made accessible in the public portal are retained by the authors and/or other copyright owners and it is a condition of accessing publications that users recognise and abide by the legal requirements associated with these rights.

- Users may download and print one copy of any publication from the public portal for the purpose of private study or research.

- You may not further distribute the material or use it for any profit-making activity or commercial gain

- You may freely distribute the URL identifying the publication in the public portal 


\title{
On-Chip Patch Antenna on InP Substrate for Short-Range Wireless Communication at $140 \mathrm{GHz}$
}

\author{
Yunfeng Dong, Tom K. Johansen, and Vitaliy Zhurbenko \\ Electromagnetic Systems, Department of Electrical Engineering \\ Technical University of Denmark \\ DK-2800 Kgs. Lyngby, Denmark \\ yundon/tkj/vz@elektro.dtu.dk
}

\begin{abstract}
This paper presents the design of an on-chip patch antenna on indium phosphide (InP) substrate for short-range wireless communication at $140 \mathrm{GHz}$. The antenna shows a simulated gain of $5.3 \mathrm{dBi}$ with $23 \%$ bandwidth at $140 \mathrm{GHz}$ and it can be used for either direct chip-to-chip communication or chip-level integration and packaging. In the transmission frequency band from $130 \mathrm{GHz}$ to $150 \mathrm{GHz}$ the estimated inband gain variation is $0.5 \mathrm{dBi}$ which guarantees gain uniformity. The antenna with optimized dimension is implemented for a transition between elevated coplanar waveguide (ECPW) and rectangular waveguide. The chip-to-waveguide transition in backto-back configuration exhibits a simulated return loss of $10 \mathrm{~dB}$ and insertion loss of $3 \mathrm{~dB}$ from $128 \mathrm{GHz}$ to $153 \mathrm{GHz}$. For higher directivity, a horn antenna is used together with the chip-towaveguide transition forming an extended packaging structure that is suitable for the transceiver ( $T x$ and $R x$ ) chips. The simulated gain of the extended packaging structure is $11.9 \mathrm{dBi}$ with $21.4 \%$ bandwidth at $140 \mathrm{GHz}$ and the in-band gain variation is $2 \mathbf{d B i}$.
\end{abstract}

\section{INTRODUCTION}

With the ever increasing demand worldwide for wireless data transmission and multimedia services, the future wireless communication systems will require higher data rate in order to provide wider bandwidth for signal transmission. With higher carrier frequency, wider bandwidth can be achieved which makes $\mathrm{THz}$ an ideal candidate for the future wireless communication systems. However, the $\mathrm{THz}$ wireless communication faces difficulties, such as low-cost compact transceiver and system packaging methodology. According to [1], $10 \mathrm{Gbit} / \mathrm{s}$ data rate can be realized by an outdoor wireless communication link at $120 \mathrm{GHz}$ over a distance of $1 \mathrm{~km}$. Besides, a fully integrated amplitude-shift keying (ASK) receiver MMIC at 300 $\mathrm{GHz}$ is reported in [2].

Fig. 1 shows the potential system diagram for fully integrated ASK transceiver MMICs based on InP double heterojunction bipolar transistor (DHBT) technology for short-range wireless communication. The carrier frequency of the system is $140 \mathrm{GHz}$ with an expected data rate of $10 \mathrm{Gbit} / \mathrm{s}$. By using ASK modulation scheme, at least $20 \mathrm{GHz}$ analog bandwidth is required by the wireless communication system for signal transmission. The antenna used for transmitting and receiving data is integrated on the transceiver chips. The challenge of designing the on-chip antenna is to achieve a high gain and at the same time provide a wideband matching from $130 \mathrm{GHz}$ to $150 \mathrm{GHz}$. By using on-chip antennas, the chip-level assembly can be avoided which simplifies the integration and packaging

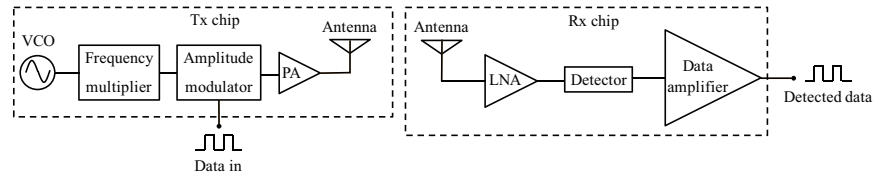

Fig. 1. System diagram for fully integrated ASK transceiver MMICs based on InP DHBT technology for short-range wireless communication.

of the transceiver chips. As a result, it provides lower loss, higher reliability, and easier fabrication especially for MMICs at $\mathrm{THz}$ frequencies.

By aligning the transceiver chips towards each other, the direct chip-to-chip communication can be realized and the assembly is not needed. When the transceiver chips are packaged, the on-chip antenna can be implemented for a chipto-waveguide transition which makes it versatile and suitable for connecting to other components and systems at $\mathrm{THz}$ frequencies. Since the transceiver chips are based on InP DHBT technology, the on-chip antenna have to be designed using the same substrate. Fig. 2 shows the substrate structure and the $50 \Omega$ ECPW. The ECPW is used for designing the transceiver chips as well as feeding the on-chip antenna. The substrate consists of a $160 \mu \mathrm{m}$ InP layer on the bottom and a $3.8 \mu \mathrm{m}$ polyimide layer on the top. The dielectric constant $\left(\epsilon_{r}\right)$ and dissipation factor $(\tan \delta)$ of polyimide are 2.9 and 0.005 , respectively. TiAuPt is used as the conductor material which is a compound of titanium, gold, and platinum. Its conductivity is $3.3 \mathrm{e} 7 \mathrm{~S} / \mathrm{m}$ and the metal thickness is $1.2 \mu \mathrm{m}$. The signal conductor layer is located on the top of polyimide while the ground conductor layer is located inside polyimide and 1.22 $\mu \mathrm{m}$ away from the top surface.

In this work, contributed to the system design, on-chip patch antenna on InP substrate is designed and analyzed by using High Frequency Structural Simulator (HFSS). The packaging structures of the designed on-chip patch antenna and the transceiver chips are addressed. In Section II, the design of on-chip patch antenna on InP substrate is described. Besides, the simulated far-field radiation pattern and gain are shown. In Section III, the rectangular waveguide packaging and assembly structure of the optimized on-chip patch antenna in back-to-back configuration are demonstrated. In Section IV, the extended packaging structure used for the potential transceiver chips is presented. The far-field radiation pattern, gain, and assembly structure of the proposed system packaging structure are explained. 


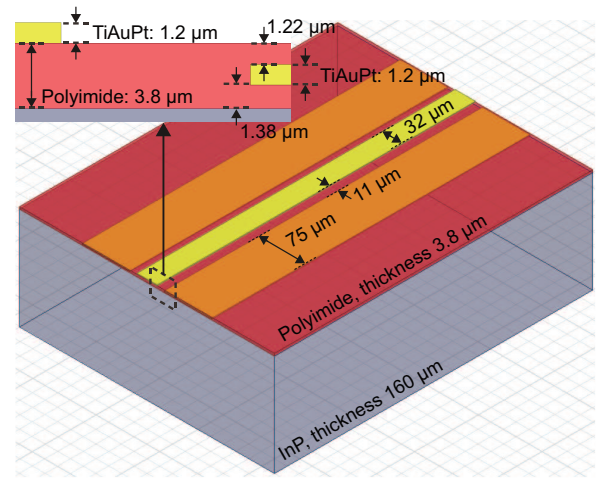

Fig. 2. Substrate structure and the ECPW used for transceiver MMIC designs.

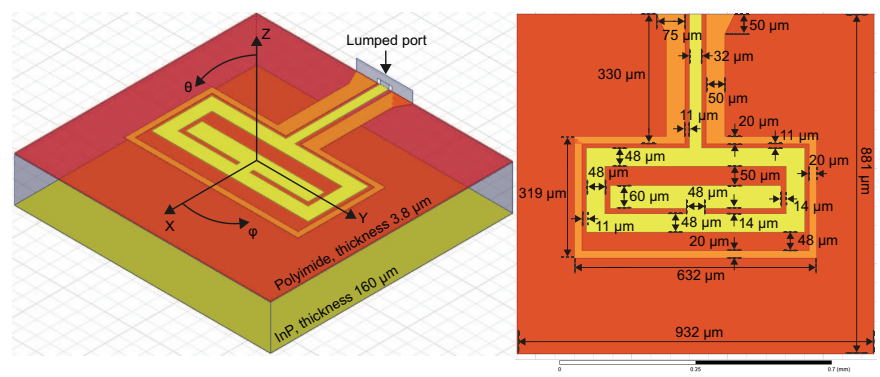

Fig. 3. Simulation structure and dimensions of the on-chip patch antenna.

\section{DESIGN OF ON-ChiP PATCH ANTENNA}

In the wireless communication system shown in Fig. 1, the antenna is used for transmitting and receiving data through free-space communication link. Patch antenna is selected to be used due to its simple planar structure and reasonable occupation area which make it suitable for on-chip integration. Besides, it can be easily fed by different types of planar transmission lines. For a conventional patch antenna design, the antenna is normally fed by microstrip line and the ground plane is on the bottom of the substrate. When a patch antenna is fed by ECPW, the patch is connected to the signal trace while the ground traces are designed either to be faded out or as a part of the patch antenna. As is shown in Fig. 2, for the $50 \Omega$ ECPW feed, the width of the signal trace is $32 \mu \mathrm{m}$ and the width of the ground trace is $75 \mu \mathrm{m}$ with a gap width of $11 \mu \mathrm{m}$.

Fig. 3 shows the simulation structure and dimensions of the on-chip patch antenna. Lumped port with vertical perfect electric conductor (PEC) bridge is used as the excitation scheme in the simulation. The PEC bridge touches the ground traces and the port is assigned to a sheet between the signal trace and the PEC bridge. The inductance of the port sheet is calibrated out from the simulation results. Another conductor layer is added on the backside of the InP substrate which works as a bottom ground plane. For simulating far-field radiation, an air box is added around the patch antenna which must be large enough reaching the far-field region of the designed on-chip patch antenna. The coordinate system for calculating far-field radiation, as is shown in Fig. 3, is located at the center of the patch geometry.

The designed on-chip patch antenna is fed by ECPW and the ground traces are tapered in from $75 \mu \mathrm{m}$ to $50 \mu \mathrm{m}$ at the beginning. The signal trace, on the top conductor layer,

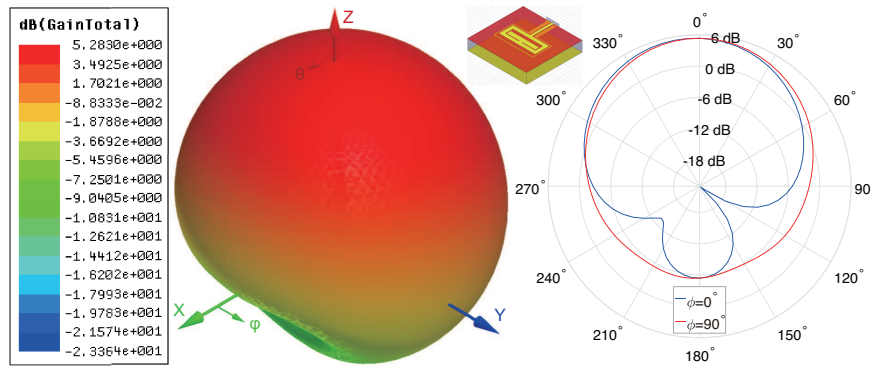

Fig. 4. Far-field radiation pattern of the on-chip patch antenna at $140 \mathrm{GHz}$.

is connected to a rectangular patch. The ground traces are tapered in from $50 \mu \mathrm{m}$ to $20 \mu \mathrm{m}$ around the patch forming a rectangular ring. In order to achieve wider bandwidth and at the same time keep the gain, the conventional rectangular patch is cut into a rectangular ring connecting to a smaller rectangular patch at the center of the patch geometry. The dimensions shown in Fig. 3 are optimized for maximum bandwidth and gain. Besides, the antenna gain in the transmission frequency band is required to be uniform in order to transmit and receive data around the same power level at different frequencies. In Fig. 4, the simulated far-field radiation pattern at $140 \mathrm{GHz}$ is shown. The maximum gain at $140 \mathrm{GHz}$ of the designed onchip patch antenna is $5.3 \mathrm{dBi}$ and it is achieved in the direction when $\theta$ and $\phi$ are $0^{\circ}$.

The designed on-chip patch antenna is simulated in terms of frequency and the results are shown in Fig. 5. The simulated return loss is better than $10 \mathrm{~dB}$ from $123.5 \mathrm{GHz}$ to $156 \mathrm{GHz}$ which represents $23 \%$ bandwidth at $140 \mathrm{GHz}$. As the transmission frequency band ranges from $130 \mathrm{GHz}$ to $150 \mathrm{GHz}$, the designed on-chip patch antenna fulfills the requirement of $20 \mathrm{GHz}$ bandwidth. In the transmission frequency band, the simulated maximum antenna gain is $5.3 \mathrm{dBi}$ while the minimum value is $4.8 \mathrm{dBi}$. A good gain uniformity is achieved since there is only $0.5 \mathrm{dBi}$ difference between the maximum and the minimum values. In comparison with the conventional rectangular patch antenna, the designed patch antenna with cutting ring structure exhibits wider bandwidth and similar gain. With integrated on-chip patch antenna, the direct chip-to-chip wireless communication can be realized between transceiver chips. The chips need to be aligned towards each other in the direction of maximum radiation and the communication system does not require any extra chip-level assembly. Antennas with high directivity are preferred for $\mathrm{THz}$ wireless communications while it is normally limited for on-chip antennas due to their planar structures. According to [3], the directivity of the onchip antenna can be increased by adding a hemisphere silicon lens on the top of the antenna which concentrates the antenna beams in the direction of wireless communication link.

\section{Packaging Structure of ON-Chip Patch ANTENNA}

The on-chip patch antenna shown in Fig. 3 is designed for direct chip-to-chip wireless communication without any packaging or measurement structures. For $\mathrm{THz}$ applications, the designed MMICs normally need to be packaged carefully in order to restrict parasitic modes, keep the bandwidth, provide good isolation from the environment, dissipate the heat generated by the circuits, and connect to other components or 

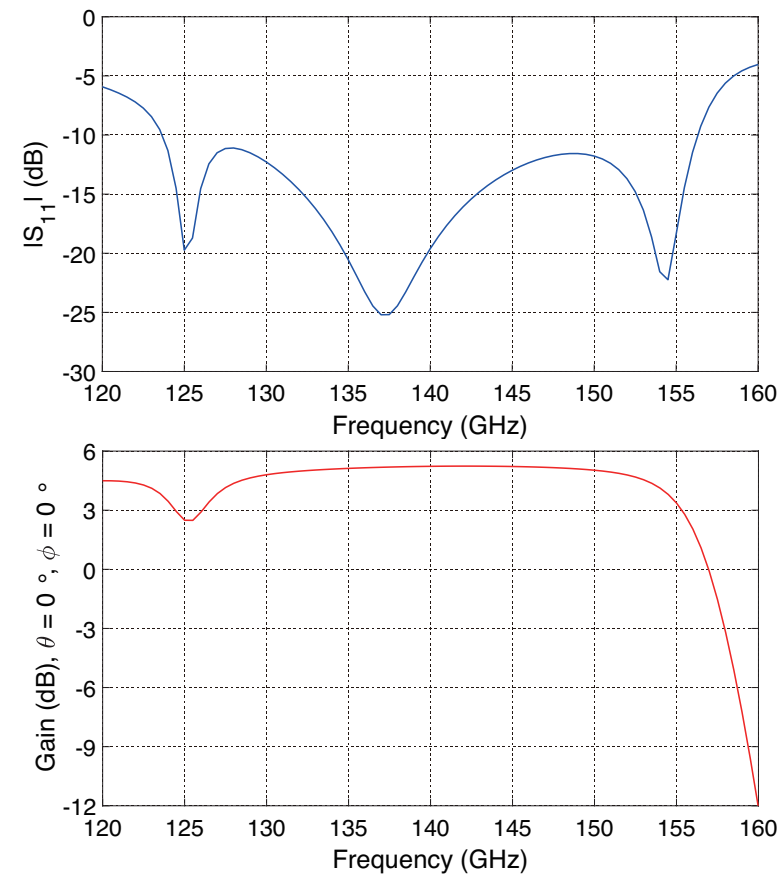

Fig. 5. Simulated return loss and gain of the on-chip patch antenna.

systems. At such high frequencies, rectangular waveguide is used as the standardized interface for connecting or cascading different circuits and components. It is also the primary type of transmission line used for guiding electromagnetic waves into and out of the circuits due to its simple structure and low loss property. Under this circumstance, the designed on-chip patch antenna can also be implemented for a chip-to-waveguide transition, in which the on-chip patch antenna is optimized and packaged by using D-band rectangular waveguide (WR$6.5,110-170 \mathrm{GHz}$ ). Fig. 6 shows the rectangular waveguide packaging structure of the optimized on-chip patch antenna in back-to-back configuration. WR-6.5 rectangular waveguide is used as the input and output of the packaging structure. The width and height of WR-6.5 rectangular waveguide are 1.651 $\mathrm{mm}$ and $0.8255 \mathrm{~mm}$, respectively. Electromagnetic waves are guided inside the rectangular waveguide and turn $90^{\circ}$ towards the direction of the maximum antenna gain. Before electromagnetic waves reaching the on-chip patch antenna, the height of the rectangular waveguide is reduced from $0.8255 \mathrm{~mm}$ to $0.52 \mathrm{~mm}$ forming a chip-to-waveguide transition which smoothly guides the electromagnetic waves from the rectangular waveguide to the on-chip patch antenna. The width of the tapered structure are kept constant and the length is 0.8 $\mathrm{mm}$. The on-chip patch antenna is located at the center of the packaging structure with an air cavity above the substrate. The height of the air cavity is $50 \mu \mathrm{m}$ with the purpose of suppressing parasitic modes.

Since the on-chip patch antenna in back-to-back configuration is packaged using rectangular waveguide inside an aluminium box, the dimensions of the on-chip patch antenna are optimized based on the new boundary conditions. As is shown in Fig. 7, two optimized on-chip patch antennas are connected by a $50 \Omega$ ECPW in the middle with a length of $860 \mu \mathrm{m}$. The length and width of the whole InP substrate are $2.214 \mathrm{~mm}$ and $0.947 \mathrm{~mm}$, respectively. It is also the typical

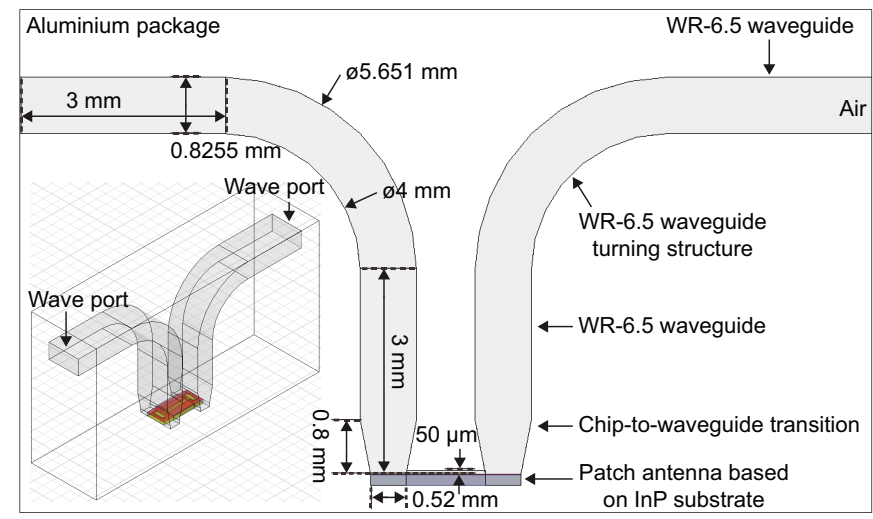

Fig. 6. Rectangular waveguide packaging structure of the optimized on-chip patch antenna.

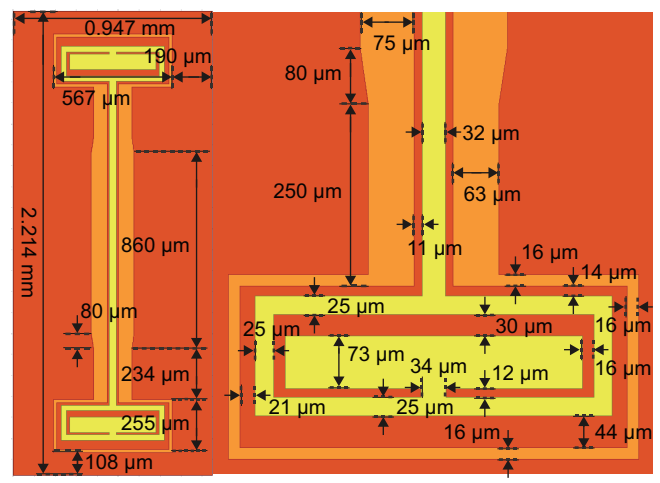

Fig. 7. Dimensions of the optimized on-chip patch antenna.

size of InP transceiver chips at $140 \mathrm{GHz}$. The length of the cavity is the same as the length of the InP substrate which means there is no gap between the end of the substrate and the wall of the cavity. It helps to suppress parasitic modes and align the InP substrate at the correct position.

Fig. 8 shows the simulation results of the chip-towaveguide transition in back-to-back configuration. Wave ports are used as the excitation scheme and they are assigned to the input and output surfaces of the packaging structure. The chipto-waveguide transition in back-to-back configuration exhibits a simulated return loss of $10 \mathrm{~dB}$ and insertion loss of $3 \mathrm{~dB}$ from $128 \mathrm{GHz}$ to $153 \mathrm{GHz}$ which covers the transmission frequency band and proves the wideband matching behavior of the optimized on-chip patch antenna. Besides on-chip antenna, E-plane probe and wire bonding can also be used for chip-towaveguide transitions [4].

In Fig. 9, the assembly structure of the chip-to-waveguide transition in back-to-back configuration is shown. The packaging structure shown in Fig. 6 is divided into 3 parts and each part can be manufactured from an aluminium box by milling. By using a spinner with a diameter of $400 \mu \mathrm{m}$, the milled cavity for InP substrate has rounded corners.

\section{Extended Packaging Structure of the System}

For higher directivity, a horn antenna is used together with the chip-to-waveguide transition forming an extended packaging structure that is suitable for the wireless communication system. Instead of direct chip-to-chip communication, 

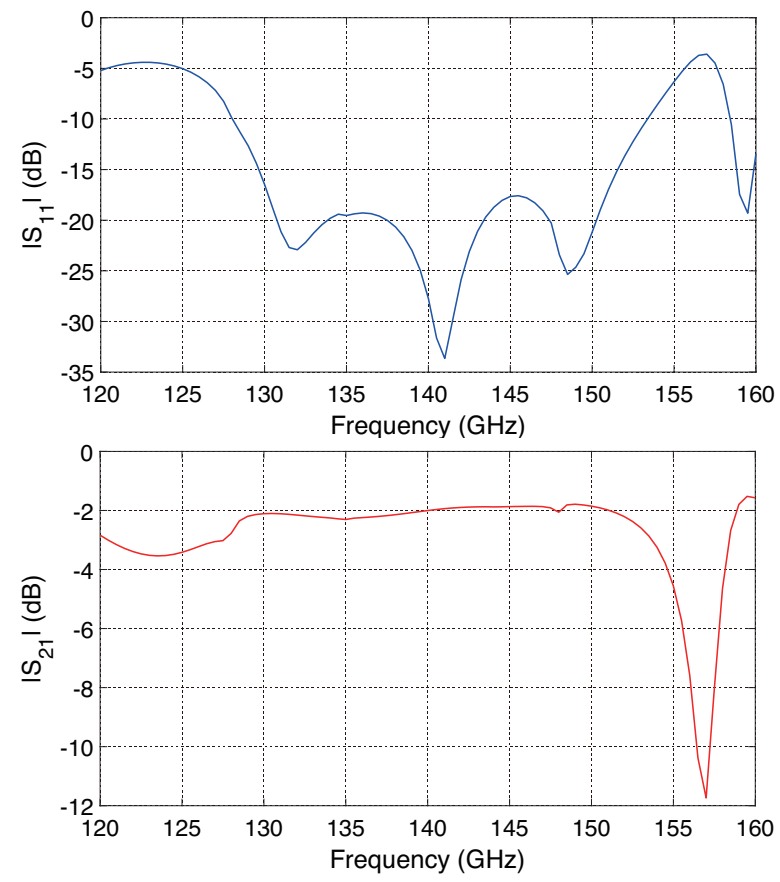

Fig. 8. Simulation results of the chip-to-waveguide transition.

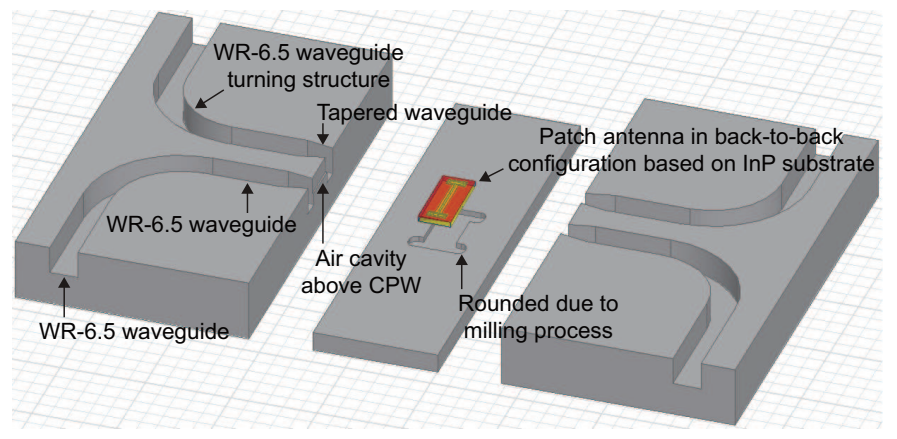

Fig. 9. Assembly structure of the chip-to-waveguide transition.

the transceiver chips are packaged which makes the system more stable and increases the directivity. Fig. 10 shows the extended packaging structure of the transceiver chips. The chip-to-waveguide transition demonstrated in Fig. 6 can be used for packaging of the transceiver chips and the on-chip patch antenna has the same dimension as it is shown in Fig. 7. The on-chip patch antenna is fed by a ECPW with a length of $370 \mu \mathrm{m}$. Besides, E-plane horn antenna is used for freespace data transmission which can be connected to rectangular waveguides and provide high antenna gain in a wide frequency range. The E-plane horn antenna is a sectoral horn flared in the direction of the E-field in the waveguide. The width and height of the antenna aperture are $1.651 \mathrm{~mm}$ and $3 \mathrm{~mm}$, respectively. The length of the E-plane horn antenna is $4 \mathrm{~mm}$.

In order to generate the correct mode at the feed point, wave port is used as the excitation scheme in the simulation and the edges of the wave port touch the ground traces of the ECPW feed. For simulating far-field radiation, an air box is added around the extended packaging structure and it is large enough reaching the far-field region. The coordinate system for calculating far-field radiation pattern, as is shown in Fig. 10,

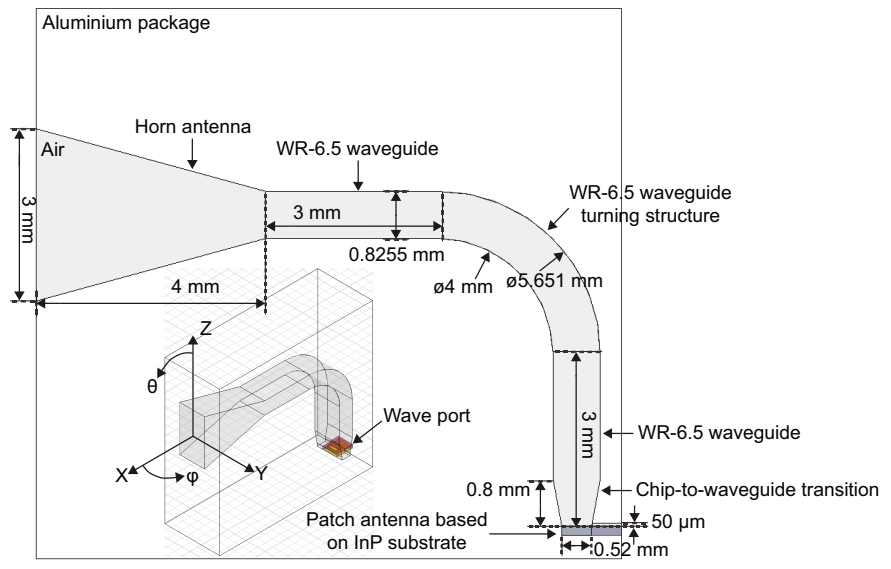

Fig. 10. Extended packaging structure of the system.

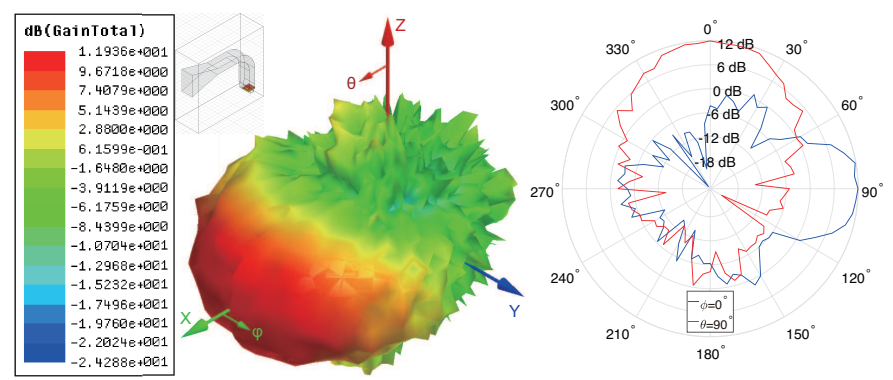

Fig. 11. Far-field radiation pattern of the extended packaging structure at $140 \mathrm{GHz}$.

is located at the center of the antenna aperture. The simulated far-field radiation pattern of the E-plane horn antenna together with the system packaging structure at $140 \mathrm{GHz}$ is shown in Fig. 11. A maximum gain of $11.9 \mathrm{dBi}$ is achieved at the direction when $\theta$ and $\phi$ are $90^{\circ}$ and $0^{\circ}$, respectively.

Fig. 12 shows the simulation results of the extended system packaging structure. The simulated return loss is better than 10 dB from $123 \mathrm{GHz}$ to $153 \mathrm{GHz}$ which covers the transmission frequency band and represents $21.4 \%$ bandwidth at $140 \mathrm{GHz}$. In the transmission frequency band, the simulated maximum gain is $12.3 \mathrm{dBi}$ while the minimum gain is $10.3 \mathrm{dBi}$. The difference between the maximum and minimum values is 2 $\mathrm{dBi}$ which guarantees a good uniformity of the data power level through a free-space communication link.

Fig. 13 shows the assembly structure of the packaged transceiver chips. The extended packaging structure shown in Fig. 10 is divided into 3 parts and each part can be manufactured from an aluminium box by milling. The onchip patch antenna, in the assembly structure, represents the transceiver chips and the cavity size needs to be adjusted when the full transceiver chips are packaged. The milled cavity has rounded corners due to the spinner whose diameter is $400 \mu \mathrm{m}$.

\section{CONCLUSION}

The design of on-chip patch antenna on InP substrate for short-range wireless communication at $140 \mathrm{GHz}$ has been presented. A chip-to-waveguide transition has been implemented by using the on-chip patch antenna with optimized dimension. Besides, an extended packaging structure is proposed for 

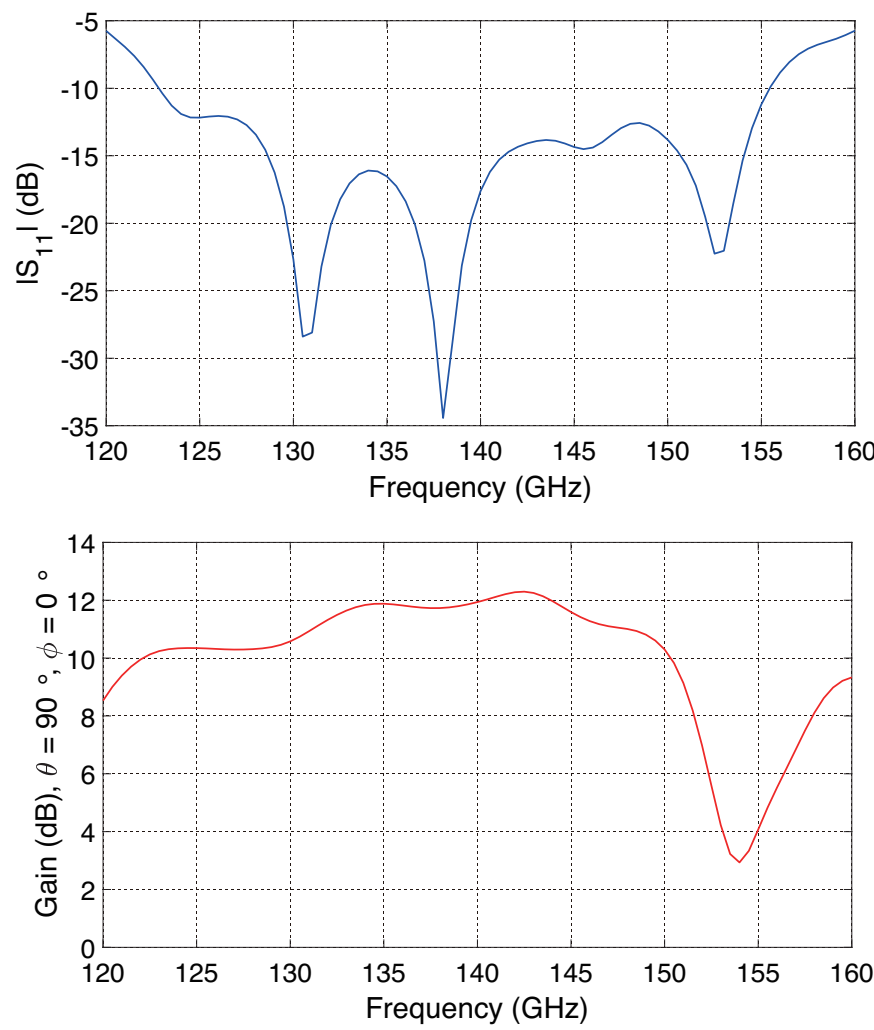

Fig. 12. Simulation results of the extended packaging structure.

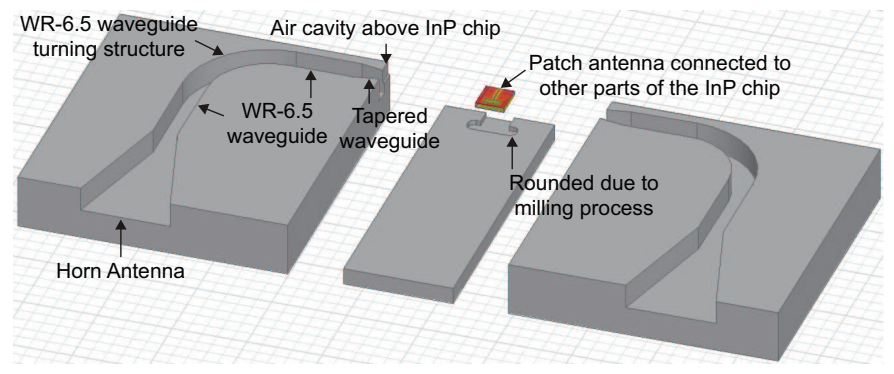

Fig. 13. Assembly structure of the extended packaging structure.

the system consisting of chip-to-waveguide transition and Eplane horn antenna. For direct chip-to-chip communication, the designed on-chip patch antenna shows a simulated gain of $5.3 \mathrm{dBi}$ with $23 \%$ bandwidth at $140 \mathrm{GHz}$. In the transmission frequency band, a good gain uniformity has been achieved. The maximum simulated gain of the designed on-chip patch antenna is $5.3 \mathrm{dBi}$ while the minimum value is $4.8 \mathrm{dBi}$. The chip-to-waveguide transition in back-to-back configuration has been demonstrated. The dimensions of the on-chip patch antenna have been optimized due to the packaging boundary conditions. The proposed packaging structure consists of WR6.5 rectangular waveguides, chip-to-waveguide transitions, and optimized on-chip patch antennas. The chip-to-waveguide transition in back-to-back configuration exhibits a simulated return loss of $10 \mathrm{~dB}$ and insertion loss of $3 \mathrm{~dB}$ from $128 \mathrm{GHz}$ to 153 $\mathrm{GHz}$. The assembly structure has been shown to prove the possibility of manufacturing the proposed packaging structure by aluminium milling. As for the system packaging of the transceiver chips, the extended packaging structure consists of on-chip patch antenna, chip-to-waveguide transition, WR-6.5 rectangular waveguide, and E-plane horn antenna. A simulated return loss of $10 \mathrm{~dB}$ has been achieved from $123 \mathrm{GHz}$ to $153 \mathrm{GHz}$ which covers the transmission frequency band and represents $21.4 \%$ bandwidth at $140 \mathrm{GHz}$. In the transmission frequency band, the maximum simulated gain is $12.3 \mathrm{dBi}$ while the minimum value is $10.3 \mathrm{dBi}$.

\section{REFERENCES}

[1] A. Hirata, T. Kosugi, H. Takahashi, J. Takeuchi, H. Togo, M. Yaita, N. Kukutsu, K. Aihara, K. Murata, Y. Sato, T. Nagatsuma, and Y. Kado. 120-GHz-Band wireless link technologies for outdoor 10-Gbit/s data transmission. Microwave Theory and Techniques, IEEE Transactions on, 60(3):881-895, Mar 2012.

[2] H. J. Song, J. Y. Kim, K. Ajito, M. Yaita, and N. Kukutsu. Fully integrated ASK receiver MMIC for terahertz communications at $300 \mathrm{GHz}$. Terahertz Science and Technology, IEEE Transactions on, 60(4):445-452, July 2013.

[3] M. Neshat, D. M. Hailu, M. R. Nezhad-Ahmadi, G. Z. Rafi, and S. Safavi-Naeini. Gain measurement of embedded on-chip antennas in $\mathrm{mmW} / \mathrm{THz}$ range. Antennas and Propagation, IEEE Transactions on, 60(5):2544-2549, May 2012.

[4] Y. Dong, T. K. Johansen, V. Zhurbenko, and P. J. Hanberg. Rectangular waveguide-to-coplanar waveguide transitions at U-band using e-plane probe and wire bonding. The 46th European Microwave Conference (EuMC), 2016 IEEE MTT-S International, 5-8, Oct 2016. 